CARNETS DE Carnets de géographes

GÉOGRAPHES.

14 | 2020

Varia

\title{
Un géographe perdu sur la piste
}

Sur les échecs (ponctuels) de l'interdisciplinarité

\section{Nicolas Canova}

\section{(2) OpenEdition}

Journals

Édition électronique

URL : https://journals.openedition.org/cdg/6138

DOI : $10.4000 /$ cdg. 6138

ISSN : 2107-7266

Éditeur

UMR 245 - CESSMA

Référence électronique

Nicolas Canova, « Un géographe perdu sur la piste », Carnets de géographes [En ligne], 14 | 2020, mis en ligne le 01 décembre 2020, consulté le 21 mai 2021. URL : http://journals.openedition.org/cdg/ 6138 ; DOI : https://doi.org/10.4000/cdg.6138

Ce document a été généré automatiquement le 21 mai 2021.

\section{(c) (1)}

La revue Carnets de géographes est mise à disposition selon les termes de la Licence Creative

Commons Attribution - Pas d'Utilisation Commerciale - Pas de Modification 4.0 International. 


\title{
Un géographe perdu sur la piste
}

Sur les échecs (ponctuels) de l'interdisciplinarité

\author{
Nicolas Canova
}

\section{Introduction}

1 L'interdisciplinarité consiste en «la mise en relation d'au moins deux disciplines, en vue d'élaborer une représentation originale d'une notion, d'une situation, d'une problématique » (Fourez, Maingain, Dufour, 2002) et suppose une « approche intégrée des problèmes " (Giolitto, Clary, 1994). Depuis l'institutionnalisation même des disciplines, un savoir scientifique se constitue ontologiquement dans une forme d'interdisciplinarité normalisée ${ }^{1}$ (Foucault, 1969). Aussi, l'interdisciplinarité est devenue pragmatique et «n'est donc pas de même nature que celle qui nourrissait les échanges des chercheurs dans les années 1970 [...]. Elle est aujourd'hui liée à des politiques de recherches qui pointent la fragmentation des milieux académiques comme un frein à l'appréhension de la complexité du monde et à l'innovation " (Endrizi, 2017: 5). L'interdisciplinarité est alors une condition souvent stricte de la constitution des programmes de recherche, notamment pour augmenter les chances de financement. On peut même soutenir que, puisqu'«à la clôture disciplinaire est opposée l'intelligence collective, [...] les problématiques "sciences et société» ringardisent les questionnements parcellaires des disciplines » (Gorga, Leresche, 2015) ${ }^{2}$.

2 Seulement, il y a dans ce propos liminaire un petit hiatus dont la problématique n'est que trop peu souvent formulée. Affichée comme gage de qualité scientifique, l'interdisciplinarité n'est toutefois pas une évidence en matière d'application et d'opérationnalité des programmes de recherche. Aussi, sa mise en œuvre pratique n'est pas aussi courante que le discours qu'elle génère peut l'affirmer; et ses petite et grande sœurs que sont la pluri- et la transdisciplinarité se confrontent aux mêmes difficultés. Faire apparaître, dans la liste d'un programme de recherche lambda, des noms de chercheurs issus de différentes disciplines (pluridisciplinarité) relève d'abord et avant tout d'un exercice rhétorique; rien ne gageant que ceux-là travailleront côte à côte dans la poursuite d'objectifs communs. Et encore plus difficile est la «mise en œuvre 
d'une méthode fondamentale commune " (Angers, Bouchard, 1992:69) à toutes les disciplines juxtaposées dans ce même programme (transdisciplinarité), sachant qu'elle est pourtant très souvent la visée finale de l'interdisciplinarité affichée.

3 L'interdisciplinarité pourrait donc être, derrière ce qu'elle représente de plus positif et d'engageant, un leurre manipulé par des acteurs de la recherche aux stratégies moins visibles et moins dicibles que celles affichées publiquement. On a pu lire ainsi qu'« elle se trouve « commandée " par les pouvoirs politiques administratifs et économiques qui contrôlent, planifient et évaluent les processus d'organisation des savoirs » (Resweber, 2011 : 183). Je livre alors dans ces Carnets de terrain une petite expérience qui illustre l'emprise que peut avoir la condition interdisciplinaire d'un programme de recherche sur son bon déroulement et les difficultés à mettre en œuvre l'interdisciplinarisation des savoirs.

\section{La meilleure façon de marcher...}

4 L'eurométropole Lille-Kortrijk-Tournai (Likoto) se définit par une longue histoire d'urbanisme horizontal, cumulant polycentrisme et discontinuité. Constituée en un groupement européen de coopération territoriale de 152 communes françaises et belges, elle est peuplée de plus de 2 millions d'habitants et couvre une surface de 3589 $\mathrm{km}^{2}$, pour une densité moyenne d'environ 550 habitants $/ \mathrm{km}^{2}$. Parmi les principaux facteurs d'étalement et de fragmentation urbaine dans cette agglomération, nous pouvons citer l'architecture de faible hauteur dominant le bâti, l'héritage industriel et ses friches et quartiers ouvriers, mais aussi les zones d'activités et commerciales plus récentes. Par leur emprise au sol facteur de dédensification, ces éléments sont constitutifs de la figure de la métropole horizontale (Vigano, 2018). Dans cet espace d'aménagement extensif, la route et le train provoquent une densification des réseaux de communication qui scinde et parcellise le territoire. Ajoutons que la métropole lilloise serait la plus ségréguée de France selon le collectif Degeyter (2017). C'est dans ce contexte qu'un groupe de chercheurs et d'acteurs lillois s'est engagé dans l'exploration des espaces publics et privés constituant les marges urbaines de Likoto.

5 Faisant suite à une recherche antérieure (Delbaere, 2015), le programme En Piste ! ${ }^{3}$ avait pour ambition d'explorer une piste évoluant le long des grandes infrastructures et voies de communication, incluant les espaces délaissés, talus et accotements, friches ou réserves, ruines et décharges ${ }^{4}$. Cette piste devait rendre compte des problématiques susmentionnées de Likoto en cherchant une continuité potentielle dans le grand nombre d'espaces définis comme des zones d'ombre de l'aménagement du territoire. Le potentiel de ces marges urbaines, interstices, non-lieux et autres tiers-espaces constituerait ainsi une trame écologique, sociale et culturelle dont la mise en politique et la planification pourraient être un objectif de conception opérationnelle porté par les participants au programme. Tel était en tout cas le postulat d'En Piste!. C'est ainsi que les équipes successives qui ont travaillé sur ce terrain ont imaginé découvrir une continuité territoriale à l'intérieur de ces espaces, qu'ils nommèrent donc «la piste ", et dont l'exploration devait permettre de conforter la réalité et l'heuristicité. Cette piste ainsi définie devait devenir un médiateur d'interdisciplinarité pour des chercheurs et acteurs issus de disciplines variées.

6 La démarche prévoyait une série de trois «randonnées scientifiques» de 3 jours chacune entre septembre 2017 et novembre 2018, «alternant des périodes d'arpentage 
et de relevés, de synthèse des relevés et de mise en débat de la méthode, puis de reformulation de cette méthode sur le terrain $»^{5}$. À cet effet, le directeur du programme avait réuni les membres de l'équipe en "collèges ${ }^{6}$ de disciplines et/ou pratiques proches. Deux grandes étapes d'arpentage étaient prévues : un travail par collège afin de statuer sur une méthodologie commune entre pairs ; puis un mélange des collèges, afin de rendre l'interdisciplinarité effective. Le programme En Piste! affichait alors une " extrême multidisciplinarité " ${ }^{7}$. En effet, « l'étude devrait dépasser ces cloisonnements disciplinaires et les postulats anthropologiques qu'ils présupposent (la distinction entre nature et culture, entre espace réel et espace projeté) $»^{8}$. La plus-value du travail devait consister principalement en la construction d'une méthode transdisciplinaire d'exploration urbaine pour saisir ces espaces délaissés de l'aménagement et qui soit transposable à d'autres terrains similaires. La proposition finale devait conduire les gestionnaires des espaces concernés à (re)considérer le bien commun que constituent les dimensions écologique, sociale et culturelle de la piste.

7 Parmi les innombrables itinéraires possibles dans Likoto, le directeur du programme avait pris le soin de tracer sur une carte deux pistes qu'il pensait cohérentes avec ses études antérieures, nous laissant le choix de l'une ou l'autre. C'est ainsi qu'un itinéraire de $60 \mathrm{~km}$ entre les communes de Lille, Marcq-en-Barœul, Croix, Lambersart et La Madeleine fut choisi ${ }^{9}$. Nous entrions alors dans une dimension constructiviste du terrain, puisque sa valeur même n'était plus supposée, mais postulée, bien que nous fut laissée toute liberté de sortir de cet itinéraire lorsque bon nous semblerait. En effet, nous allions vite nous rendre compte que la piste n'en était pas une stricto sensu et donc qu'une définition préalable allait brouiller nos pistes de recherches.

8 À la réunion de cadrage, je lançais alors mes idées premières quant à ce que je pouvais essayer de faire pour répondre aux attentes du programme :

-Je discuterais au mieux avec les membres de mon «collège sociologique» tel que l'avait constitué le projet (une sociologue et trois géographes). J'attendais ici beaucoup d'une rencontre 1/ avec une sociologue, car j'espérais faire des entretiens sur la piste que j'avais imaginée objet de pratiques, bien que furtives, marginales et/ou illicites 2/ avec deux autres géographes que je savais quantitativistes, avec qui il me fallait trouver des points d'accroche infra-disciplinaire, étant plutôt qualitativiste.

- Dans une première phase d'observation et de description, je noterais, enregistrerais et photographierais la présence d'objets et de traces qui révèlent une présence humaine. Vues comme les marques d'une fixation des usages, sortes de cairns, je noterais comment s'ancrent des pratiques circulatoires dans l'espace. Je partirais chemin faisant en relevant les tags, graffs et gravures, repérant des paysages sonores et inventoriant d'autres indices: feux éteints, canettes, mégots, préservatifs, déchets... qui me rapprocheraient du paradigme indiciaire $^{10}$ de Ginzburg (1980). En effet, ce bagage théorique m'apparaissait particulièrement intéressant pour enquêter, suivre une piste incertaine, en suivant une série d'indices révélateurs de ses géographies.

\section{Du terrain au terrain, sans passer par la théorie}

9 Le premier point d'achoppement a eu lieu lors de la réunion de lancement qui ne donna aucune consigne et se limita presque exclusivement aux détails pratiques. Aussi, l'absence de la sociologue, finalement indisponible, marqua le premier décalage entre la multidisciplinarité affichée et l'effectif finalement réduit du quorum en SHS. Voilà 
qui limitait mon travail de préparation de la première randonnée ; travail tout autant freiné par un attentisme général.

10 J'arrivais donc au premier séjour de terrain en espérant trouver un moment de mise en place d'un protocole de recherche lors de la demi-journée prévue à cet effet. C'est là que je compris réellement que ma pratique de chercheur ne coïncidait pas avec ce que l'on attendait de moi. En effet, l'ensemble des programmes de recherche auxquels j'avais pu participer jusque-là se fondait, assez classiquement, sur le triptyque problématique-hypothèse-méthode issu à la fois d'un état de l'art conséquent et de plusieurs terrains susceptibles de fournir les données nécessaires pour y répondre. Dans le cas d'En Piste!, il n'en fut rien. Être livré à moi-même sur un terrain sans protocole établi me mit dans une posture expectative inédite, car l'objet n'était pas non plus de faire remonter du terrain un quelconque fait généralisable ou une information spécifique. Il nous était surtout demandé de confronter nos méthodes entre nous. D'ailleurs, le terrain avait été déjà bien exploré par les programmes qui nous précédaient et dont plusieurs membres étaient parmi nous. Considérant que le couple inductif/déductif «exclut de la réflexion sur la construction du savoir disciplinaire l'ensemble des médiations et significations liées à une pratique / expérience à dimension spatiale ${ }^{11}$, nous devions le dépasser pour, dès le départ, imaginer les capacités d'hybridation de nos pratiques respectives. Mais sans méthodologie, nos méthodes (re)devenaient des pratiques. Notre posture de chercheur se mêlait à celle d'usagers. En effet, sans protocole, qu'est-ce qui devait différencier, par exemple, la prise photographique du naturaliste, du géographe et de l'habitant? Le dessin de l'artiste, du paysagiste, du technicien ou du gestionnaire? Comment distinguer dans ces conditions nos attaches disciplinaires?

11 Désormais, nous n'étions plus que trois à composer le collège devenu exclusivement "géographique». Tandis qu'un seul d'entre nous avait pris l'initiative d'une méthode systématique $^{12}$, nous discutions de nos observations en marchant le long de cette piste incertaine. Rien ne nous permettait pour l'instant d'établir des conclusions, même provisoires, en dehors d'une acceptation commune de la description qui nous avait été faite des espaces marginaux de Likoto. Un problème de taille se posait toutefois : les rares individus que nous croisions ne semblaient pas suivre la direction que nous demandait de suivre le terrain préalablement sélectionné. La piste devenait chaque fois plus fictive que les tronçons et autres lieux qui la composaient devenaient des espaces indépendants : terrains vagues, dessous de ponts, voies ferrées désaffectées ou actives, carrefours et ronds-points, lotissements, quartiers divers, décharges, talus d'autoroute, friches industrielles, ancienne gare de triage. Rien ne semblait indiquer qu'une continuité prévalait sur la fragmentation dans cet ensemble métropolitain. Et peu de signes permettaient de donner du sens à une potentielle action collective de réunion de ces espaces éparpillés, éclatés par des forces, champs et vitesses distinctes. Avec nos pratiques plurielles relativisant nos attachements disciplinaires, c'était notre terrain lui-même qui se dérobait sous nos pieds.

12 Alors que nous débriefions tous ensemble en intérieur, je commençais à protester: «Mais que doit-on faire? Que cherche-t-on? Et puis, cette piste, n'est-elle pas inventée? Quand va-t-on travailler l'interdisciplinarité? ». Tout en conservant l'idée que les objectifs du programme étaient louables et que la démarche globale pour les atteindre me semblait aussi innovante que techniquement habile, je doutais fortement de nos (mes) capacités à alimenter en contenu les discussions avec une telle ligne 
méthodologique. Aussi, je demandais que le mélange des collèges se fasse plus vite et espérais voir ce que pouvaient bien faire les autres. Notons alors que les deux urbanistes inscrits dans le collège "aménagiste " ne furent finalement pas engagés dans la recherche. Le responsable d'une association d'usagers-cyclistes listé dans le collège " gestionnaire et usagers » s'est quant à lui rapidement dégagé du programme. La nouvelle marche programmée fut plus enrichissante scientifiquement. Je m'intéressais notamment au « dessin rapide » d'un paysagiste ou au "diagnostic flash » du naturaliste. La première pratique définit des croquis effectués à la hâte à chaque nouvelle ouverture paysagère ou point de vue jugé pertinent par l'auteur. La seconde consiste à délimiter des espaces restreints (quelques mètres carrés) dans chaque géotope arpenté (sentiers, friches industrielles, embroussaillement, voies ferrées désaffectées ou actives, accotements du réseau routier) afin d'en saisir la biodiversité et plus spécifiquement les espèces remarquables dans un but exploratoire et/ou statistique. Leurs atouts étaient principalement de ne pas trop ralentir la marche collective et ainsi de permettre une activité considérée comme disciplinaire dans une dynamique de groupe.

Lors du deuxième séjour, nous participions tous ensemble à une grande marche collective. Bien que soudés et tous volontaires, le terrain nous mit à l'épreuve (passages boueux, en bord d'autoroute, dans une friche végétale dense, franchissement de grillage, de voie ferrée active). Cela aurait pu, selon les hypothèses du programme, augmenter la nécessité de nos échanges. Mais aucun partage de compétences n'œuvrait réellement. D'abord, je constatais que les discussions entre " pistards » tournaient plus autour des représentations subjectives de la piste que des pratiques, méthodes ou théories qui permettaient de l'objectiver. Si cela démontrait le fort potentiel du terrain à nourrir les pratiques et imaginaires des uns et des autres, cette situation nous éloignait toujours un peu plus des attendus du projet en matière de construction d'une méthodologie commune. Chacun était à sa place sans vraiment prendre celle de l'autre. Le paysagiste, par exemple, n'appuyait que très peu son dessin rapide sur les commentaires de l'équipe. Il en était de même pour le géographe ayant adopté une démarche systématique qui resta inchangée, même si sa production fut partagée et questionnée par le groupe au moment d'en imaginer la restitution. Ce fut également le cas du naturaliste. Une des artistes prit quant à elle le parti pris de "l'affût", qui consista à se fixer dans un lieu pour y produire un travail particulier (peinture). Isolé de fait de la démarche collective, cela permit toutefois de rencontrer un jardinier « sauvage » et des graffeurs que nous présentons ci-dessous.

De mon côté, j'orientais la recherche sur la pratique sociale du terrain. La rencontre de deux riverains fit l'objet d'un enregistrement. Ils nous révélèrent surtout que le morceau de piste que nous explorions à côté de chez eux était absent de leurs préoccupations. Une frontière nette délimitait l'espace de leur pratique de celui que nous lui avions accolé. Trois autres découvertes, qui donnèrent lieu à une série de photographies inédites (figure 1) étaient également des indices intéressants à suivre en termes de pratiques sociales. Un « sentier » de préservatifs usagés laissant présager un spot de prostitution, un «champ " d'excréments tout autour d'un camp de gens du voyage ou encore un "charnier» de laboratoire de cannabis indiquaient la valeur ponctuelle de ces lieux que leur attribuaient certaines pratiques urbaines. Mais ils achevaient de déconstruire la conception linéaire du terrain et avec elle l'idée de piste, du fait d'une absence de continuité entre chacun d'entre eux, et ce malgré leur 
proximité. La spontanéité de ces espaces, sortant de tout cadre prescriptif orienté par des actions aménagistes en termes tout du moins de pratiques sociales, se calquait donc plus sur le modèle de la zone que sur celui de la trame.

Figure 1. Échantillon des 3 séries photographiques de terrain

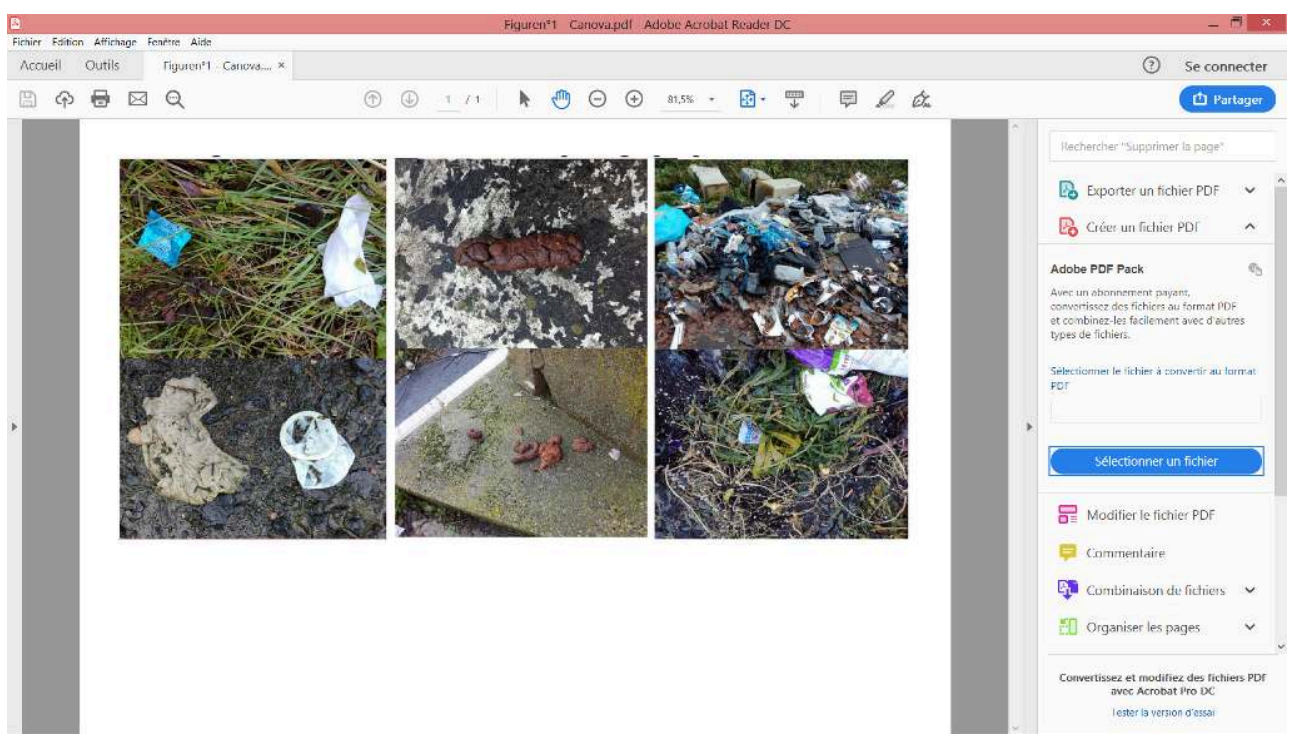

Sources : auteur, septembre 2017

J'eus donc également la chance de rencontrer (avec le groupe) un voisin dont un entretien collectif fit émerger l'intervention spontanée de jardinier "sauvage» (défrichement, plantation, élagage) dans le lieu nommé « la cathédrale $»^{13}$. Il constitue un bon exemple de l'attention et du soin que les habitants sont susceptibles de porter aux espaces déqualifiés par l'aménagement. Je fis aussi la rencontre de deux graffeurs venus marquer l'espace de leur signature sous l'acronyme Handstyle. Lycéens venus ici pour la première fois, ils encourageaient la lecture d'une distribution ponctuelle des pratiques sociales qui devait l'emporter sur l'hypothèse de linéarité. Ces modalités d'appropriation des lieux constitutifs de la piste et les pratiques spatiales qui en découlent permettraient aussi d'avancer sur les actions possibles à mener sur notre terrain. En ce sens, j'acceptais que « se restreindre à la visée cognitive et à la dimension méthodologique ne permet[tait] pas d'envisager la richesse et la complexité du terrain. L'éventail de ses dimensions recouvre à la fois les champs de la méthodologie, de la pratique, de l'expérience, de la représentation, etc., champs que le chercheur investit de significations dans une tension entre habitus disciplinaire, action militante et projet de vie $»^{14}$. À partir de ces indices de fréquentation (préservatifs, graffs, détritus, excréments et autres «traces»), j'aurais pu adopter la méthode des transects perpendiculaires à la piste; coupant ainsi l'espace en gradients ou sections d'aménagement, réfléchissant par seuils, ruptures, coupes. Mais cela revenait à travailler sur l'appréhension du terrain en oubliant les objectifs d'interdisciplinarité qui, bien que premiers dans la rédaction du projet, n'étaient toujours pas à l'agenda.

17 Arrivé au bout de cette seconde randonnée, je décidais alors de questionner le groupe sur ses objectifs lors de petits entretiens. Ces derniers avaient un rôle exploratoire pour développer la réflexion sur l'engagement disciplinaire et les perspectives interdisciplinaires de chacun. Les questions portaient principalement sur nos capacités 
à répondre aux objectifs interdisciplinaires du programme. J'y questionnais l'ancrage disciplinaire de chacun (formations, métiers, pratiques, méthodes, références scientifiques) et le positionnement par rapport aux autres collègues.

Il en ressortit une variété de positions en lien à la "catégorie » disciplinaire dans laquelle mes collègues avaient été classés au préalable. Les discours captés ici sont saisissants quant aux différences et aux proximités des personnes entre elles, rien ne rapprochant les chercheurs de même discipline, ou de disciplines proches. Par exemple, à la question "à qui ressembles-tu le plus? ", le paysagiste qui utilise essentiellement le dessin et le texte renvoie au gestionnaire des espaces verts qui a axé sa pratique sur l'écoute ornithologique et la production de petites capsules vidéo mettant en scène notre exploration. Ce dernier dit être éloigné de la pratique des paysagistes, trop orientée vers l'artificialisation de la nature. Autre exemple, plus de la moitié de l'équipe interrogée s'est dite proche du géographe quantitativiste ayant adopté la méthode systématique, pourtant seul chercheur impliqué dans une telle démarche avec le naturaliste ; qui lui ne s'est pas reconnu dans ce géographe. Pour ce qui est des raisons de l'ouverture du dialogue interdisciplinaire, les réponses sont en grande majorité orientées vers la convivialité du lieu de résidence ${ }^{15}$ et la souplesse de la direction du programme ${ }^{16}$. À l'unanimité, à la question « que nous reste-t-il à faire pour atteindre les objectifs d'interdisciplinarité ?", les réponses affirment la nécessité d'une production commune. Cette production prendra la forme d'un site Internet au sein duquel sont regroupées les productions individuelles et collectives ${ }^{17}$.

Aucune conclusion toutefois ne peut en ressortir en l'état, mais cette phase exploratoire fut intéressante pour préparer un travail plus approfondi sur la place, supposée importante, des représentations et imaginaires disciplinaires dans la formulation des prédicats théoriques et méthodologiques de la recherche. La place de ma discipline de rattachement est ici révélatrice de ce constat, puisque les trois géographes présents ont choisi trois manières de faire. La construction des catégories disciplinaires est encore une fois soumise à la représentation que les autres s'en font.

\section{Vers d'autres pistes}

Une absence remarquée de références, spécifiquement bibliographiques et issues des recherches sur l'interdisciplinarité, dans le projet initial comme dans l'ensemble des discussions orales ou écrites me semble être la principale raison de cet échec relatif. Elle peut laisser penser que l'on ne se réfère pas ici à la science comme une démarche collective hétérogène, mais à une science pratiquée en circuit fermé qui fait abstraction d'un corpus ayant abordé ces questions. J'ai aussi été surpris que tout le bagage psychogéographique, dont la plupart des concepts sont encore bien présents dans les écoles d'architecture et de paysage (dérive, déambulation, robinsonnade, transect) mais surtout dont la théorie-pratique est inscrite au cœur même du projet En Piste!, ne soit pas mieux pris en compte. Nous avons pourtant pu voir en quoi elle était capable de minimiser les inerties disciplinaires et de décloisonner des savoirs (Coverley, 2011; Canova, 2015).

21 Aussi, les conclusions inscrites au rapport de synthèse du programme se sont arrêtées sur trois plus-values de cette recherche: la capacité de la marche à favoriser la production interdisciplinaire par le temps et l'espace qu'elle propose aux rapports intersubjectifs ; la possibilité offerte aux disciplines de dialoguer par les outils que sont 
le dessin, la photographie, la cartographie et l'interprétation paysagère ; la difficulté à tenir le postulat d'une linéarité spontanée et cohérente des délaissées urbains de Likoto. Si elles sont alors qualifiées de provisoires, c'est parce qu'elles n'ont pas réussi à faire consensus et à déboucher sur une écriture collective dans un rapport de synthèse. Tout l'enjeu de notre problématique prend corps ici.

La présentation des résultats sur le site Internet dédié montre notre capacité à explorer individuellement et collectivement le terrain. Nous avons en cela bien adopté une démarche pluridisciplinaire. Mais la difficulté réside dans notre incapacité à dépasser cette juxtaposition de "résultats " pour en faire une synthèse interdisciplinaire. Cela marque l'incapacité du collectif à faire référence, en dehors de ses expériences individuelles, à l'altérité de sa démarche et à l'ailleurs du terrain; c'est-à-dire à s'extraire de sa pratique "habituelle ", à rendre reproductible une méthode élaborée collectivement et, se faisant, à s'inscrire dans la dimension collective de la production scientifique. En témoigne le seul article émanant d'En Piste! (Lemoine, 2018), fruit d'une seule personne dont la démarche de terrain et de «laboratoire » fut principalement individuelle et infra-disciplinaire.

Le parti pris de ne pas faire intervenir de référence n'était pourtant pas une posture affirmée de la recherche, puisque pendant les discussions chacun a mobilisé ses références et qu'un cadrage théorique introduisait le texte du projet. De mes lectures sur le sujet, j'avais surtout dégagé trois angles problématiques : la différence nécessaire entre pluri-, inter- et transdisciplinarité qui fonde trois contextes scientifiques de coopération distincts ; la difficulté de définir strictement les contours d'une discipline dans la pratique, et particulièrement la géographie; les risques d'inertie auxquels peuvent conduire l'inscription disciplinaire et plus spécifiquement sa découpe en souschamps tels que pensés généralement en épistémologie. Aucune de ces questions n'a pu être traitée par l'équipe. Voilà principalement en quoi un programme qui se base explicitement et principalement sur un affichage de l'interdisciplinarité pour obtenir un financement n'est pas en mesure d'avancer des orientions méthodologiques et protocolaires pour répondre à sa problématique; ni de mettre en œuvre dans son déroulé les actions nécessaires à la réalisation de cette condition de financement.

Mais peut-être que cela aura permis une meilleure mise en dialogue des participants ainsi qu'un gain de temps sur le lancement du programme et son déroulement technique. C'est finalement là que les ambitions du projet pourraient être revues $a$ minima: ne pas penser la disciplinarité comme un fait, mais comme un construit épistémologique. La recherche des coopérations, croisements et hybridations potentielles entre les différentes démarches représentées ne sont pas des ouvertures pluri-, inter- ou transdisciplinaires, mais relèvent alors plutôt d'un principe intersubjectif au sens où Habermas (1973) l'a critiqué ; c'est-à-dire en soustrayant toute idéologie prépondérante à la production commune issue de la rencontre de plusieurs êtres de pensée autonome. Objectiver les savoirs et méthodes pour poser les bases de l'interdisciplinarité relève ainsi plus d'une pratique que d'une théorie. C'est d'ailleurs ce que nous aurons pu conclure collectivement, lorsque l'on lit dans notre rapport que «les échanges spontanés sont la forme minimale d'articulation interdisciplinaire que génère la marche ». Une catégorisation des pratiques rapportées par cette expérience montre qu'il y a différents degrés d'agir ensemble au sein du programme. L'observation, et ses conséquences sur l'amorce de transferts de compétences, de savoirs et parfois 
d'«identité » scientifique ; marquerait notre posture pluridisciplinaire. La coopération dans le processus d'exploration urbaine qui tente un protocole commun définit les contours d'une interdisciplinarité d'action. Finalement, les essais de coproduction amorcent la transdisciplinarité de nos pratiques, même s'ils se sont contenus dans l'oralité. La synthèse de ces trois postures imaginées par le déroulement du programme reste toutefois à « inventer».

Il faut ici préciser deux choses importantes pour ne pas mettre en défaut l'action globale de l'équipe d'En Piste!. D'abord, dans le cadre de la recherche-action propre à certaines écoles d'architecture et de paysage, l'utilité sociale des programmes légitime des sorties de piste quant à l'académisme et aux protocoles scientifiques. Ce n'est pas le sujet de cet article. Aussi, il y a dans ce que notre collègue naturaliste a su nommer «transdisciplinarité rêvée » à propos de notre pratique, toute la bonne intention d'une recherche en lien à ce que l'on pourrait qualifier d'imaginaire interdisciplinaire. Tout en voulant exprimer ici l'essai louable que constituait En Piste! en matière de croisement heuristique des disciplines, en faire la critique a pour principal objectif que de travailler, encore et toujours, à l'amélioration des conditions de la recherche.

Pour revenir sur ma vision des programmes de recherche, qui s'est construite dans une pratique assez simple et récurrente en SHS, que l'on résume souvent au leitmotiv «rechercher c'est se poser les bonnes questions»; il est vrai qu'elle a montré ses limites. Mais ne travailler que "depuis» une réalité, sans opérer d'allers et retours avec la théorie me rendrait bien incapable d'imaginer les contours d'une telle science ainsi produite, certainement trop internalisante. C'est en cela que la démarche itérative est susceptible d'intervenir dans la modification de notre problématique, pour que le terrain ne soit pas l'unique point de départ.

Suivant Resweber (2011: 189) lorsqu'il affirme que "l'interdisciplinarité fait partie intégrante de la constitution de toute discipline ", nous pourrions nous demander in fine si l'intégration des savoirs, connaissances et expériences ne serait donc pas une problématique qui peut s'émanciper d'une réflexion purement disciplinaire. Mieux, n'y a-t-il pas ici une condition même de l'émancipation des chercheurs des carcans disciplinaires que leur imposent certains de leurs collègues et plus largement les cadres institutionnels ? Je garde toutefois à l'esprit que, outre la sympathique atmosphère du programme En Piste! et l'heureuse expérience des rencontres que j'y fis, il nous servit aussi à questionner l'interdisciplinarité en ce qu'elle « contribue, de manière implicite, à définir la manière même dont une discipline organise et révise ses méthodes et ses concepts. En la renvoyant du cadre où elle s'est fixée au champ dans lequel elle s'est inscrite et de ce champ au milieu recouvrant l'inter-champ où elle s'est enracinée, elle l'invite à refaire le chemin même de sa genèse » (Resweber, 2011 : 173). Pour le reste, pas besoin de l'invoquer comme le messie de l'appréhension nouvelle des objets complexes de la recherche contemporaine. Mieux que des "disciplines", ce sont des savoirs, connaissances et expériences réunies qui s'imposent à leur appréhension (Morin, 1977). 


\section{BIBLIOGRAPHIE}

ANGERS P., BOUCHARD C. (1992), «L'intégration, source de l'interdisciplinarité », in Delisle R., Begin P. (dir.), L'interdisciplinarité au primaire, une voie d'avenir?, Sherbrooke, Éditions du CRP, pp. 69-77.

CANOVA N. (2015), «Penser l'espace avec ses pieds », EspacesTemps.net [En ligne : https:// www.espacestemps.net/articles/penser-lespace-avec-ses-pieds/].

COVERLEY M. (dir.) (2011), Psychogéographie! Poétique de l'exploration urbaine, Lyon, Les Moutons Électriques.

DEGEYTER COLLECTIF (2017), Sociologie de Lille, La Découverte, Paris.

DELBAERE D. (dir.), 2015, En marge. Paysage et biodiversité des accotements infrastructurels de l'Eurométropole Lille-Kortrijk-Tournai, Rapport de synthèse, ministère de l'Écologie, région NordPas-de-Calais, conseil départemental du Nord et Métropole européenne de Lille.

ENDRIZZI L (2017), L'avenir de l'université est-il interdisciplinaire ?, Dossier de veille de l'IFÉ no. 120, Lyon, ENS de Lyon.

FOUCAULT M., (1969), L’archéologie du savoir, Paris, Tel-Gallimard.

FOUREZ G., MAINGAIN A., DUFOUR B. (dir.), (2002), Approches didactiques de l'interdisciplinarité, Bruxelles, De Boeck.

GINZBURG C. (1980), « Signes, traces, pistes. Racines d'un paradigme de l'indice », Le Débat, novembre, pp. 3-44.

GIOLITTO P., CLARY M. (1994), Éduquer à l'environnement, Paris, Hachette éducation.

GORGA A., LERESCHE J.-P. (2015), Disciplines académiques en transformation : entre innovation et résistance, Paris, Éditions des archives contemporaines.

HABERMAS J. (1973), La technique et la science comme idéologie, Denoël/Gonthier, Paris.

LEMOINE, G. (2018), « Un diagnostic botanique “en marchant” peut-il apporter des données représentatives des enjeux relatifs à la flore et aux habitats des bordures des infrastructures de transport ? », Bulletin de la Société Botanique du Nord de la France, no. 71, vol. 1-4, pp. 29-30.

MORIN E. (1977), La méthode, 1. La Nature de la Nature, Paris, Le Seuil.

RESWEBER J-P. (2011), « Les enjeux de l'interdisciplinarité », Questions de communication, no. 19, pp. 171-200.

VIGANO P. (2018), « Préface : Dans la métropole horizontale », in Léger J-M., Mariolle B. (Dir.), Densifier / Dédensifier. Penser les campagnes urbaines, Marseille, Ed. Parenthèses.

\section{NOTES}

1. Michel Foucault (1969) considère que « les sciences humaines se développent à partir de trois disciplines-souches : la biologie, l'économie et la linguistique ». Plus généralement, il incite à penser dans son Archéologie du savoir, que la survie des disciplines se fonde sur un principe d'interdisciplinarité permanent, principalement dû à l'évolution des concepts échangés, produits, et consommés continuellement entre les disciplines. 
2. Cités par Endrizi (2017: 5).

3. Financé par le programme ITTECOP - voir http://www.ittecop.fr/

4. Une courte cartographie animée présente le parcours de la piste sur https://la-piste-delikoto.org/presentation-de-la-piste-de-likoto/

5. Présentation du projet sur http://www.ittecop.fr/images/docman-files/docman-files/rapportfinal/rapport-ittecop-apr-2017/fiche-projet/19_Ittecop_ENPISTE.pdf

6. 5 collèges furent constitués pour un total de 15 chercheurs : « écologue » (2), « sociologique » (3), « aménagiste » (3), « artistique » (2), « gestionnaire » (2) et « usagers » (3).

7. Delbaere D., in https://urbanwastelands.sciencesconf.org/browse/author?authorid=567093

8. Ibidem

9. Pour une description voir : https://la-piste-de-likoto.org/presentation-de-la-piste-de-likoto/

10. Proposition d'application épistémologique réunissant des méthodes de diagnostic en psychologie clinicienne et les préceptes de l'enquête du roman policier principalement. Carlo Ginzburg a suggéré que le paradigme indiciaire «aidera peut-être à sortir des impasses de l'opposition entre « rationalisme » et « irrationalisme » » (Ginzburg, $1980: 3$ ).

11. "Les dimensions du terrain en géographie ", Appel à contribution, Calenda, Publié le dimanche 15 avril 2007, https://calenda.org/192975

12. Prises photographiques aux quatre points cardinaux tous les cent mètres en vue d'une analyse paysagère.

13. Lieu proche de la maison où nous résidions tous, descriptible comme une vaste friche sous le tablier d'un pont autoroutier, délimitée par une voie rapide en contrebas et longée par une voie ferrée d'un côté, des habitations de l'autre côté; la hauteur des piliers du pont nous rappelait alors l'architecture d'une cathédrale.

14. Présentation du projet sur http://www.ittecop.fr/images/docman-files/docman-files/ rapport-final/rapport-ittecop-apr-2017/fiche-projet/19_Ittecop_ENPISTE.pdf

15. Tous en «résidence» chez Bertrand, que je remercie pour son accueil et ses repas inoubliables.

16. Résident principalement dans l'absence de protocole méthodologique imposé.

17. https://la-piste-de-likoto.org/

\section{RÉSUMÉS}

L'affichage interdisciplinaire est inhérent aux programmes de recherche contemporains. Pourtant, l'interdisciplinarité n'est pas une donnée factuelle ni une méthode applicable à toute recherche et pourrait même être un leurre. Je questionne alors dans ce papier l'emprise que peut avoir la condition interdisciplinaire d'un programme de recherche sur son bon déroulement et les difficultés à mettre en œuvre l'interdisciplinarisation des savoirs à laquelle il prétend se confronter. Pour ce faire, voici une petite archéologie de ma participation au programme En Piste!, qui espérait contribuer à définir les contours interdisciplinaires d'une méthodologie d'exploration urbaine.

Interdisciplinary display is inherent to most contemporary research programs. However, interdisciplinarity is neither a factual data nor a method applicable to any research. It could even be a lure. In this article, I question some influences of the interdisciplinary condition of a specific 
research program, on its progress and the difficulties it faces in facing the interdisciplinarisation of knowledge to which it claims to contribute. Here is a brief archaeology of my participation in the program En Piste! that, which aimed at defining the interdisciplinarity contours of an urban exploration methodology.

INDEX

Thèmes : Carnets de terrain

Mots-clés : interdisciplinarité, programme de recherche, retour d'expérience, exploration urbaine.

Keywords : Interdisciplinarity, Research Program, Feedback, Urban Exploration.

\section{AUTEUR}

\section{NICOLAS CANOVA}

LACTH - ENSAP de Lille

n-canova[at]

lille.archi.fr 